\title{
CRITERIA FOR SUCCESSFUL WEANING FROM MECHANICAL VENTILATION IN CHILDREN
}

\author{
Olha Filyk ${ }^{1}$ \\ ${ }^{1} 1$ Department of Anesthesiology and Intensive Care, Danylo Halytsky Lviv National Medical University, Lviv, Ukraine \\ filyk_olha@meduniv.lviv.ua \\ ORCID: http://orcid.org/0000-0003-3160-7617
}

ARTICLE INFO

Article history:

Received date 07.07.2020

Accepted date 28.07.2020

Published date 31.08.2020

Section:

Technologies and equipment of clinical medicine

DOI

$10.21303 / 2313-8416.2020 .001395$

KEYWORDS

weaning

mechanical ventilation

children
ABSTRACT

The aim of the research was to establish criteria for successful weaning from mechanical ventilation in children based on analysis of Paediatric rapid shallow breathing index, maximum amplitude of diaphragm movements, diaphragm thickening fraction and pressure support (PS), which ensure minimal respiratory muscle load, level of consciousness which ensure minimal respiratory muscle load and presence of cough and swallowing reflexes and previous unsuccessful attempts of weaning.

Investigated problem: there is no consensus on the basic physiological parameters for successful extubation that have to be achieved during weaning from mechanical ventilation in children due to variability in size and degree of maturity of lungs and patients' comorbidities. It leads to the lack of clinical justification for the routine practice of weaning in children.

The main scientific results: We have established a list of causes of unsuccessful weaning depending on the function of the diaphragm in children with different types of respiratory failure.

We have clarified and supplemented the list of reasons for unsuccessful weaning from mechanical ventilation depending on nutritional status and level of serum electrolytes in children.

We have identified and supplemented the list of reasons for unsuccessful weaning from mechanical ventilation depending on the disorders of neurological status in children.

We have supplemented the algorithm for predicting difficult weaning from mechanical ventilation in children.

The area of practical use of the research results: the obtained results have to increase the rate of successful weaning in children with acute respiratory failure in pediatric intensive care units.

(C) The Author(s) 2020. This is an open access article under the CC BY license http://creativecommons.org/licenses/by/4.0).

\section{Introduction}

\section{1. The object of research}

The object of research was to establish criteria for successful weaning from mechanical ventilation in children based on analysis of Paediatric rapid shallow breathing index, maximum amplitude of diaphragm movements, diaphragm thickening fraction and pressure support (PS), which ensure minimal respiratory muscle load and level of consciousness, presence of cough and swallowing reflexes and previous unsuccessful attempts of weaning.

\section{2 Problem description}

Acute respiratory failure is one of the most common cause of death among children in pediatric intensive care units (PICU) [1]. No matter what is the etiology of this syndrome, from $30 \%$ to $64 \%$ of children in PICU need mechanical ventilation (MV), and unsuccessful weaning might be present in $6.2 \%$ [2] to $36 \%[3,4]$ of them. Both the early start of weaning and its delay are harmful to patient. Early extubation can lead to catastrophic circulatory or respiratory disorders, and long-term mechanical ventilation with high parameters in $29-80 \%$ of patients is associated with atrophy and dysfunction of diaphragm [5]. Last decade represents us not only animal models, which illustrate how diaphragm activity during MV support can attenuate ventilator-induced diaphragmatic dysfunction [6], but also human studies which confirm that length of MV is associated with the degree of diaphragm atrophy [7]. 
There is also important to identify and prevent potential routes of diaphragmatic injury with providing diaphragm-protective strategy of mechanical ventilation. In addition, there is no consensus on the basic physiological parameters for successful extubation that have to be achieved during weaning from mechanical ventilation in children due to variability in size and degree of maturity of lungs and patients' comorbidities. It leads to the lack of clinical justification for the routine practice of weaning in children.

\section{3. Suggested solution to the problem.}

The described problem can be solved with making the analysis of criteria which ensure minimal respiratory muscles load during weaning from MV. The working hypothesis was that Paediatric rapid shallow breathing index, maximum amplitude of diaphragm movements, diaphragm thickening fraction and pressure support (PS) can not predict results of weaning from MV in children.

\section{Materials and Methods}

We conducted a prospective cohort single-center study at the Department of Anesthesiology and Intensive Care at Lviv Regional Children's Clinical Hospital "OHMATDYT” from January 2018 till April 2020. We included patients with acute respiratory failure who was mechanically ventilated for more than 3 days. Exclusion criteria for the study were: the refusal of the patient's legal representatives to participate in the study at any of its stages, the patient's agonizing state upon admission, and the onset of MV less than $48 \mathrm{~h}$ after prior weaning.

The study included 89 patients aged 1 month - 18 years. All patients were randomly divided into 2 groups (using random.org). Group I included patients who received lung-protective ventilation strategy, group II - patients who received diaphragm-protective in addition to lung-protective ventilation strategy. 82 patients were included in the data analysis. We studied indicators of diaphragm function (amplitude of diaphragm movement, thickening fraction and it was considered that decreasing of this indicator less than $15 \%$ was a marker of diaphragm weakness; increasing it up to $35 \%$ and more was a marker of high respiratory function and a potentially damaging factor for diaphragm), acid-base balance changes and parameters of MV (spontaneous respiratory rate, total minute volume ventilation and spontaneous minute volume ventilation and it comparison with total minute volume ventilation, minimal pressure support, which was need for absence respiratory muscles overload), Paediatric rapid shallow breathing index, Glasgow coma scale level, presence of cough and swallowing reflexes and previous unsuccessful attempts of weaning.

To assess age-dependent data, patients were divided into age subgroups: 1st subgroup children 1 month - 1 year; 2nd subgroup - children 1-3 years; 3rd subgroup - children 3-6 years; 4th subgroup - children 6-13 years; 5th subgroup - children 13-18 years.

Stages of the study: 1st day (d1), 3rd day (d3), 5th day (d5), 7th day (d7), 9th day (d9), 14th day (d14), 28th day (d28).

The primary endpoint was the duration of weaning from MV. Secondary endpoints were trends in Paediatric rapid shallow breathing index and rate of complications: frequency of reintubations and tracheostomies, prolonged mechanical ventilation, death (we assessed the presence of these adverse events daily from the time of inclusion of the patient in the study, 28 days after clinical, laboratory and instrumental signs of acute respiratory failure till discharging patient from the hospital).

\section{Results}

We found that restoration of spontaneous breathing occurred significantly faster in II group in all age subgroups of patients in comparison with the I group. It was confirmed by a significantly faster increasing of rate of spontaneous breathing in II group. These indicators were (Fig. 1) for the I and II groups at stage $\mathrm{d} 1-4[3 ; 5]$ breath/min and $6[5 ; 7]$ breaths $/ \mathrm{min}(\mathrm{p}=0.12)$, at stage $\mathrm{d} 3 \mathrm{in}$ II group data were in 2 times higher than in I group $(16[14 ; 18]$ breaths $/ \mathrm{min}$ and $8[6 ; 9]$ breaths/min, $\mathrm{p}=0.05)$. At stage d7 and $\mathrm{d} 9$ in II group they were $22[20 ; 24]$ breaths $/ \mathrm{min}$ and $24[21 ; 28]$ breaths/min, in comparison with $14[13 ; 15]$ breaths/min and $16[14 ; 17]$ breaths/min in I study group ( $\mathrm{p}=0.01$ and $\mathrm{p}=0.04)$ 


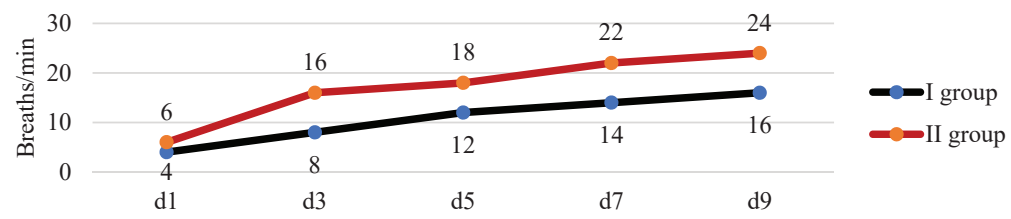

Fig. 1. Changes of spontaneous respiration rate during MV in $1^{\text {st }}$ age subgroup

The spontaneous minute volume ventilation in 1st age subgroup of patients was significantly higher in II group at stages d3, d7 and d9 in 2 times, 2.5 times and 2.1 times in comparison with I group $(\mathrm{p}=0.05 ; \mathrm{p}=0.002$ and $\mathrm{p}=0.002)$.

In $2^{\text {nd }}$ age subgroup (Fig. 2) in II group at the stage d1 the frequency of spontaneous breaths was $16[6 ; 18]$ breaths/min in comparison with $6[2 ; 10]$ breaths/min in group I $(p=0.04)$; at stage d3 in group II this parameter increased to $25[12 ; 26]$ breaths/min, in comparison with $9[5,14]$ breaths/min in group $I(p=0.02)$. Further to stage $d 7$, the frequency of spontaneous breaths did not increase significantly in group II, while in group I this tendency to a gradual increasing was maintained.

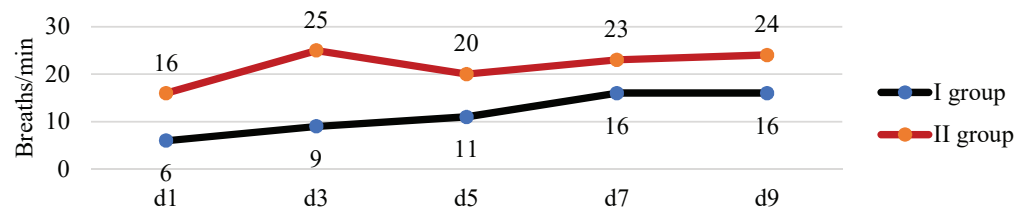

Fig. 2. Changes of spontaneous respiration rate during $M V$ in $2^{\text {nd }}$ age subgroup

The minute volume of spontaneous ventilation was significantly different in both groups of patients from the stage $\mathrm{d} 3$, when in group I it was $1.0[0.65 ; 1,225] \mathrm{l} / \mathrm{min}$, in comparison with $1.9[1,885 ; 2.12] 1 / \mathrm{min}(\mathrm{p}=0.02)$ in group II; at stage $\mathrm{d} 5$ these indicators were $0.964[0.845 ; 1.12]$ $1 / \mathrm{min}$ in I group and $2.2[2.14 ; 2.45] 1 / \mathrm{min}$ in II group $(p=0.001)$. This shows the restoration of proper function of spontaneous respiration pattern and active coordinated work of respiratory muscles. At the stage $\mathrm{d} 7$, the minute volume of spontaneous ventilation in II group was in 1.6 times higher than in I group $(2.55$ [2.43; 2,855] 1/min in II group and 1,554 [1,16; 1.942] 1/min in I group ( $p=0.04)$.

In the $3^{\text {rd }}$ age subgroup (Fig. 3), the frequency of spontaneous respiration was significantly higher in II group and at stage d5 was 29 [20; 30] breaths/min in group II, and $14[11 ; 16]$ breaths/min in group I $(\mathrm{p}=0.02)$; at stage $\mathrm{d} 7-25[22 ; 27]$ breaths/min in comparison with $14[12 ; 18]$ breaths/min $(p=0.04)$ and at stage $\mathrm{d} 9-21[19 ; 25]$ breaths/min in comparison with $12[10 ; 13]$ breaths $/ \min (\mathrm{p}=0.02)$.

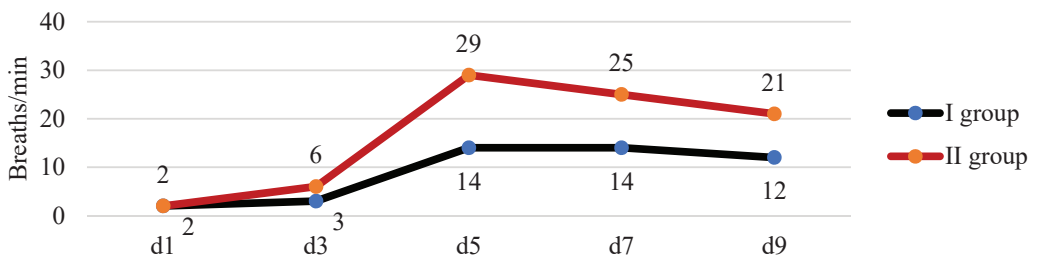

Fig. 3. Changes of spontaneous respiration rate during MV in $3^{\text {rd }}$ age subgroup

Among patients of the 3 rd age subgroup, the volume of spontaneous ventilation significantly faster increased in II group from stage $\mathrm{d} 3$ to stage $\mathrm{d} 9$ of patients, in comparison with I group.

The frequency of spontaneous respiration in $4^{\text {th }}$ subgroup (Fig. 4) in II group at stage $\mathrm{d} 3$ was $8[6 ; 12]$ breaths $/ \mathrm{min}$, and $0[0 ; 4]$ breaths $/ \min (\mathrm{p}=0.02)$ in I group; at $\mathrm{d} 5-8[5 ; 10]$ breaths $/ \mathrm{min}$ and $0[0 ; 5]$ breaths $/ \min (p=0.01)$, at stages $\mathrm{d} 7$ and $\mathrm{d} 9$ in group I increased to $5[4 ; 8]$ breaths $/ \mathrm{min}$ and returned to $3[2 ; 6]$ breaths $/ \mathrm{min}$, while in group II was $9[5 ; 10]$ breaths/min and $12[4 ; 14]$ breaths/min $(\mathrm{p}=0.05$ and $\mathrm{p}=0.001)$, respectively.

Significant differences were found in the data of spontaneous minute volume ventilation in $4^{\text {th }}$ age subgroup: it was almost absent on stage $d 5$ in I group and gradually increased from stage d1 to stage d5 in II group. 


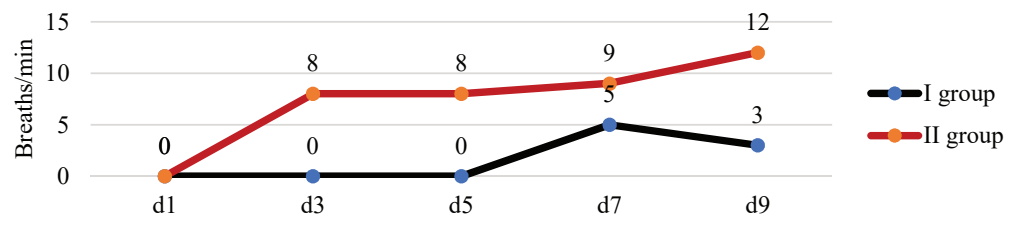

Fig. 4. Changes of spontaneous respiration rate during MV in $4^{\text {th }}$ age subgroup

In the fifth age subgroup (Fig. 5) there were significantly faster increase in spontaneous minute volume ventilation due to both the frequency of spontaneous breaths and, apparently, an increase in lung compliance in patients of II group, in comparison with I group from stage $\mathrm{d} 3$ to stage $\mathrm{d} 7$. Thus, already at stage $\mathrm{d} 3$ spontaneous minute volume ventilation was $1.93[1.645 ; 2.97] 1 / \mathrm{min}$ in group II, and 0.76 [0; 1.59] $1 / \mathrm{min}$ in group I ( $\mathrm{p}=0.04)$; at stage d5 increased to $3.735[0,293 ; 7,695] \mathrm{l} / \mathrm{min}$ in group II and up to $2.4[0.255 ; 2.84] \mathrm{l} / \mathrm{min}$ in group I ( $\mathrm{p}=0.05)$; at the stage $\mathrm{d} 7$ it was $4[3.3 ; 4.51] \mathrm{l} / \mathrm{min}$ in group II, and $2.6[0.985 ; 2.96] \mathrm{l} / \mathrm{min}$ in group I $(\mathrm{p}=0.02)$.

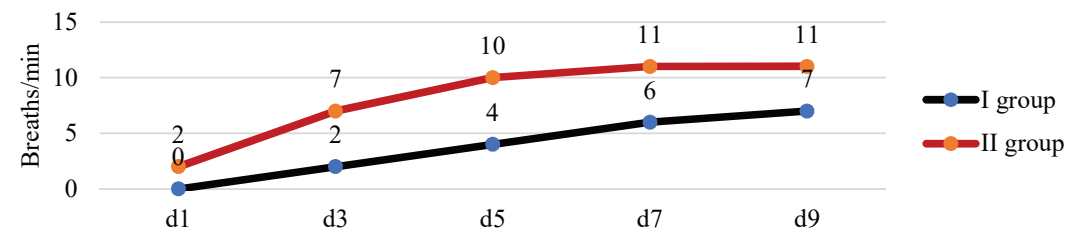

Fig. 5. Changes of spontaneous respiration rate during $M V$ in $5^{\text {th }}$ age subgroup

Summarizing all the above, spontaneous minute volume ventilation in II group of patients in all age subgroups increased significantly highly and faster in comparison with patients in I group. Moreover, in the II group readiness for weaning from mechanical ventilation as a presence of $75 \%$ and more of spontaneous minute volume ventilation to the total minute volume ventilation was achieved in 1st age subgroup - at d7 stage; in 2nd age subgroup - at the d3 stage, in 3rd age subgroup - at d5 stage; in the 4th and 5th age subgroups - only at stage d9; while in I group among all age subgroups of patients this indicator was not achieved even to step $\mathrm{d} 9$.

We identified age-specific features of diaphragm dysfunction during MV: in patients of the first age subgroup in group I there were found weakness for the right hemidiaphragm with compensatory excessive level of work for the left dome at the beginning of weaning and at stage d9, while in group II diaphragm overload was registered only at stage d5. In patients of 2nd age subgroup in group I changes were the opposite to described previous - we found excessive work of the right hemidiaphragm with low contractions of left dome at all stages of study in I group, while in II group - the only episode of diaphragmatic weakness in stage $\mathrm{d} 3$. In the 3rd age subgroup the proper diaphragmatic activity in I group of patients was restored later than in II group. In the 4th age subgroup in I group there was episode of high work of a diaphragm at stage d5, whereas in II group - all data of diaphragm function were within the recommended parameters for diaphragm-protective strategy of MV at all stages of our study. In the 5th age subgroup in I group excessive work of both right and left domes of diaphragm was significantly more often registered during weaning than in II group, however, in II group were found episodes of both type changes diaphragmatic weakness and excessive work.

We studied the presence of cough and swallowing reflexes in patients of both groups and obtained the following results: among patients of group I at study stages d1, d3, d5, these indicators were $75.3 \%, 79 \%, 82.7 \%$, and $83.9 \%$, and from the stage of the study $\mathrm{d} 7$ they did not change were $83.9 \%$. In II patients group these data were $80 \%(\mathrm{p}=0.28)$ at stage $\mathrm{d} 1 ; 85.9 \%(\mathrm{p}=0.12)$ on $\mathrm{d} 3$, $87.3 \%(\mathrm{p}=0.23)$ on $\mathrm{d} 5$; and $88.7 \%$ on $\mathrm{d} 7(\mathrm{p}=0.41)$.

It was found that combination of such indicators as Paediatric rapid shallow breathing in$\operatorname{dex}>6.2$ breaths $/ \mathrm{min} / \mathrm{ml} / \mathrm{kg}(\mathrm{AUC}=0.739,95 \% \mathrm{CI}=0.618-0.861 ; \mathrm{p}=0.001)$ with $70 \%$ sensitivity and $79.1 \%$ specificity for successful extubation), amplitude of the diaphragm movement less than $8 \mathrm{~mm}$, fraction of diaphragm thickening less than $15 \%$ or more than $35 \%$, tidal volume less than $4.5 \mathrm{ml} / \mathrm{kg}$, PS more than $12 \mathrm{~cm} \mathrm{H}_{2} \mathrm{O}$ to achieve minimal respiratory system load, no spontaneous cough reflex, less than 11 points according to Glasgow coma scale, and two or more previous- 
ly unsuccessful weaning attempts give us unfavourable prognosis for weaning from mechanical ventilation. In addition, it was found that for patients with hypoxemic and combined hypercapnic-hypoxemic type of acute respiratory failure in the presence of bulbar disorders and inability to swallow liquid food, the prognosis for weaning was favourable in case of securing lower airways with a cuffed tube with maintenance pressure in a cuff not less than $15-20 \mathrm{~mm} \mathrm{Hg}$.

\section{Discussion}

It is known that muscle atrophy frequently occurs in children admitted to ICU [10] and diaphragmatic with other skeletal muscle atrophy and weakness are associated with difficulty weaning from MV [5].

However, historically adequacy of spontaneous ventilation is checked only during spontaneous breathing trial test, via T-tube or some different devices, and it gives the only confirmation of patients' readiness to be weaned from MV on time of extubation [11]. We suggest observing spontaneous respirations adequacy all the time since we stop sedation and gradually decrease support parameters of MV. It will improve our clinical judgment how to wean this patient as soon as possible and safe.

We also know, that it is recommended that all children on respiratory support preferably should breathe spontaneously, with the exception of the most severely ill child with obstructive airway (strong agreement), restrictive (strong agreement) or mixed disease (strong agreement) requiring very high ventilator settings and intermittent neuromuscular blockade (strong agreement) [12]. Therefore, good diaphragmatic activity [8] with providing enough level of spontaneous minute volume ventilation [9] have to improve clinical outcomes in mechanical ventilated children. However, it is unknown the sufficient spontaneous respirations rate to achieve it.

Our study identified possible mechanisms of spontaneous breathing inadequacy and the causes of unsuccessful weaning from mechanical ventilation in children in accordance to diaphragm function and rate of spontaneous respirations.

It was found that the recovery of spontaneous respiration occurred significantly faster in the II study group of all age subgroups of patients compared with the I study group, which is confirmed by a significantly faster increase in spontaneous respiration rate, significantly faster growth of spontaneous minute volume ventilation among patients of the II group of all age subgroups, faster achievement of readiness for weaning due to the presence of $75 \%$ of spontaneous minute volume ventilation (among children of the 2nd age subgroup it was achieved already at the stage $\mathrm{d} 3$, among patients of the 3rd age group - at the stage d5; among patients of the 1st age group - at the stage of $\mathrm{d} 7$; in the 4th and 5th age subgroups only at stage $\mathrm{d}$, while in study group I among all age subgroups of patients was not reached to stage d9). We found that in II group at stages $\mathrm{d} 3$ and $\mathrm{d} 5$ were low levels of thickening fraction of right hemidiaphragm (less than $15 \%$ ) with a frequency of their detection of $78 \%$ at stage $\mathrm{d} 3$ and $59 \%$ at stage d5, compared with $32 \%$ and $17 \%$ at similar stages among patients of study I group. Signs of excessive contraction of the right dome of the diaphragm (thickening fraction over $45-50 \%$ ) were in $67 \%$ of patients in group I and only in $5 \%$ patients in group II at the stage $\mathrm{d} 7(\mathrm{p}=0.0001)$, which is the evidence of excessive diaphragm work in I group.

It was found that it was reduced the duration of mechanical ventilation: in patients of the 1 st age subgroup by 1.5 times $(p=0.08)$; in patients of 2 nd age subgroup - by 2.4 times $(p=0.18)$; in 4 th age subgroup - by 1.75 times $(\mathrm{p}=0.1)$; in 5 th age subgroup - by 4.25 times $(\mathrm{p}=0.009)$. In $\mathrm{pa}-$ tients of 3rd age subgroup duration of mechanical ventilation increased by 1.1 times $(p=0.68)$. The frequency of complications (reintubations) was reduced in 1st age subgroup by 4.3 times $(\mathrm{p}=0.02)$; in 2 nd age subgroup - by 3.4 times $(p=0.04)$. There were no significant differences in the frequency of tracheostomy among patients of I and II groups.

\section{Conclusion}

Amplitude of diaphragm movement over $8 \mathrm{~mm}$ with fraction of diaphragm thickening 15-35\% provide enough spontaneous minute volume ventilation in mechanically ventilated children. Moreover, pressure support over $12 \mathrm{~cm} \mathrm{H}_{2} \mathrm{O}$ allows to achieve minimal respiratory system load and Paediatric rapid shallow breathing index $>6.2$ breaths $/ \mathrm{min} / \mathrm{ml} / \mathrm{kg}$ might be criterion for successful weaning. In addition, it is still needed to have cough reflex and more than 11 points according to Glasgow Come Scale to be successfully weaned. 


\section{References}

[1] Burns, J. P., Sellers, D. E., Meyer, E. C., Lewis-Newby, M., Truog, R. D. (2014). Epidemiology of Death in the PICU at Five U.S. Teaching Hospitals*. Critical Care Medicine, 42 (9), 2101-2108. doi: http://doi.org/10.1097/ccm.0000000000000498

[2] Dres, M., Jung, B., Molinari, N., Manna, F., Dubé, B.-P., Chanques, G. et. al. (2019). Respective contribution of intensive care unit-acquired limb muscle and severe diaphragm weakness on weaning outcome and mortality: a post hoc analysis of two cohorts. Critical Care, 23 (1). doi: http://doi.org/10.1186/s13054-019-2650-z

[3] Abdo, M., Talat, M., Zamzam, S. (2014). Difficult weaning from mechanical ventilation in the pediatric ICU. Ain-Shams Journal of Anaesthesiology, 7 (1), 76-79. doi: http://doi.org/10.4103/1687-7934.128423

[4] Fontela, P. S., Piva, J. P., Garcia, P. C., Bered, P. L., Zilles, K. (2005). Risk factors for extubation failure in mechanically ventilated pediatric patients. Pediatric Critical Care Medicine, 6 (2), 166-170. doi: http://doi.org/10.1097/01. pcc.0000154922.65189.48

[5] Goligher, E. C., Dres, M., Fan, E., Rubenfeld, G. D., Scales, D. C., Herridge, M. S. et. al. (2018). Mechanical Ventilationinduced Diaphragm Atrophy Strongly Impacts Clinical Outcomes. American Journal of Respiratory and Critical Care Medicine, 197 (2), 204-213. doi: http://doi.org/10.1164/rccm.201703-0536oc

[6] Martin, A. D., Joseph, A.-M., Beaver, T. M., Smith, B. K., Martin, T. D., Berg, K. et. al. (2014). Effect of Intermittent Phrenic Nerve Stimulation During Cardiothoracic Surgery on Mitochondrial Respiration in the Human Diaphragm*. Critical Care Medicine, 42 (2), e152-e156. doi: http://doi.org/10.1097/ccm.0b013e3182a63fdf

[7] Schepens, T., Verbrugghe, W., Dams, K., Corthouts, B., Parizel, P. M., Jorens, P. G. (2015). The course of diaphragm atrophy in ventilated patients assessed with ultrasound: a longitudinal cohort study. Critical Care, 19 (1). doi: http://doi.org/ 10.1186/s13054-015-1141-0

[8] Johnson, R. W., Ng, K. W. P., Dietz, A. R., Hartman, M. E., Baty, J. D., Hasan, N. et. al. (2018). Muscle atrophy in mechanically-ventilated critically ill children. PLOS ONE, 13 (12), e0207720. doi: http://doi.org/10.1371/journal.pone.0207720

[9] Smallwood, C. D. (2019). Spontaneous Breathing Trials in Children: Putting the "T" in SBT. Respiratory Care, 64 (5), 614-615. doi: http://doi.org/10.4187/respcare.07057

[10] Kneyber, M. C. J., de Luca, D., Calderini, E., Jarreau, P.-H., Javouhey, E. et. al. (2017). Recommendations for mechanical ventilation of critically ill children from the Paediatric Mechanical Ventilation Consensus Conference (PEMVECC). Intensive Care Medicine, 43 (12), 1764-1780. doi: http://doi.org/10.1007/s00134-017-4920-z

[11] Schepens, T., Dres, M., Heunks, L., Goligher, E. C. (2019). Diaphragm-protective mechanical ventilation. Current Opinion in Critical Care, 25 (1), 77-85. doi: http://doi.org/10.1097/mcc.0000000000000578

[12] Schepens, T., Dianti, J. (2020). Diaphragm protection. Current Opinion in Critical Care, 26 (1), 35-40. doi: http://doi.org/ $10.1097 / \mathrm{mcc} .0000000000000683$ 